Case Report

\title{
Rhizopus Laryngitis Post Allogenic Stem Cell Transplantation
}

\author{
${ }^{1}$ Mayank Dhamija, ${ }^{2}$ Sanjeev Kumar Sharma, ${ }^{2}$ Dharma Choudhary, ${ }^{2}$ Vipin Khandelwal, \\ ${ }^{2}$ Sweta Kothari, ${ }^{2}$ Divya Doval, ${ }^{2}$ Gaurav Kharya, ${ }^{3}$ Purabi Barman and ${ }^{4}$ Ramlingam WVBS \\ ${ }^{I}$ Department of Pediatrics, Division of Hematology Oncology, The Hospital for Sick Children, Toronto, Canada \\ ${ }^{2}$ Department of Hemato-Oncology and Bone Marrow Transplantation, \\ ${ }^{3}$ Department of Microbiology, \\ ${ }^{4}$ Department of Oto-Rhino-Laryngology, \\ BLK Super Speciality Hospital, 5, Pusa Road, New Delhi, India, Postal code 110005, India
}

Article history

Received: 16-03-2016

Revised: $18-05-2016$

Accepted: 04-06-2016

Corresponding Author: Mayank Dhamija

Department of Pediatrics,

Division of Hematology

Oncology, The Hospital for

Sick Children, Toronto, Canada

Tell: +1 647-527-5334

Email:dr.mayankdhamija@gmail.com Mayank.dhamija@sickkids.ca

\begin{abstract}
Invasive Fungal Infections (IFI) are an important complication and constitute significantly to mortality and morbidity, post Hematopoietic Stem Cell Transplant (HSCT). We report a very rare case of fungal laryngitis post HSCT in a 15-year-old girl with Thalassemia major. She received Busulphan, Cyclophosphamide and Antithymocyte Globulin (Bu-Cy-ATG) based conditioning pre Matched Sibling Donor (MSD) transplant and presented on day 10 post-transplant with hoarseness of voice progressing to stridor and respiratory obstruction. CT neck revealed laryngeal edema and she was ultimately diagnosed, by a laryngoscopy biopsy, histopathology and fungal culture to be having invasive fungal laryngitis due to Rhizopus. She responded partially to combination antifungal treatment with amphotericin B and posaconazole, family refused for laryngectomy and she was discharged with a tracheostomy tube in situ. Patient eventually succumbed due to bacterial sepsis. IFI should be considered in the differential diagnosis of unexplained symptoms post HSCT as timely intervention and treatment can save a lot of morbidity and mortality.
\end{abstract}

Keywords: Amphotericin B, Mucormycosis, Rhizopus, Laryngitis

\section{Introduction}

Invasive fungal infections constitute one of the predominant infections in HSCT recipients resulting in high morbidity and mortality. With the use of fluconazole prophylaxis, the incidence of candida infection has decreased significantly but the incidence of aspergillus and mucormycosis has increased (Marr et al., $2000 ; 2002)$. These infections are difficult to diagnose in the setting of cytopenias post stem cell transplant (Sharma et al., 2013). We present a case of fungal laryngeal chondritis caused by rhizopus in a patient with thalassemia major post allogenic HSCT, which is the first such case reported.

\section{Materials and Methods}

A 15-year-old female, known case of thalassemia major, on regular transfusions since the age of 11 months, received matched sibling donor allogenic HSCT. She was on chelation with desferrioxamine, her pre transplant serum ferritin was $2528 \mathrm{ng} \mathrm{mL}^{-1}$ and liver biopsy revealed hemosiderosis (score 4/4) and liver fibrosis (Ishak stage 3). She received $\mathrm{Bu}-\mathrm{Cy}-$ ATG based myeloablative conditioning and was infused peripherally harvested stem cells from the donor (CD 34 positive stem cell count $2.07 \times 10^{6} / \mathrm{kg}$ ). Graft versus Host Disease (GvHD) prophylaxis included methotrexate and cyclosporine. Anti-fungal and anti-viral prophylaxis included fluconazole and valacyclovir respectively. Neutrophils engrafted on day +11 and platelets engrafted on day +18 posttransplant. On day +10 post HSCT, patient developed mild hoarseness of voice, which progressed to stridor and difficulty in breathing. Computed tomography of neck and chest revealed diffuse edema of the glottis and supraglottic soft tissue causing significant luminal compromise of the larynx and supraglottic airway (Fig. 1). There was no evidence of any other disease in chest and paranasal sinuses were normal. She was treated with intravenous steroids. 


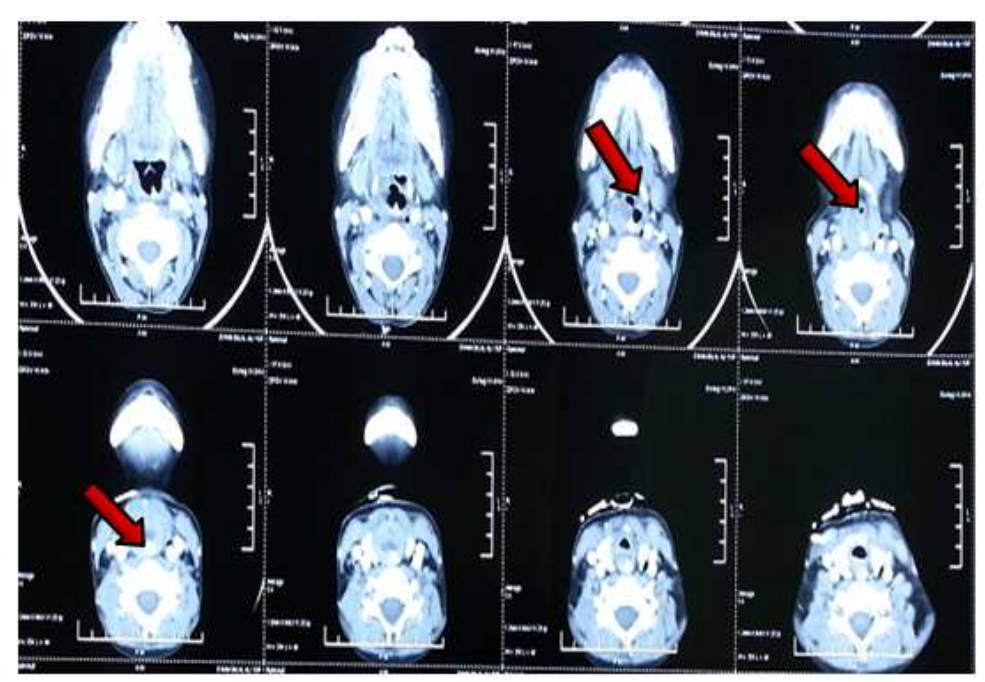

Fig. 1. Serial sections of CT scan of the neck showing diffuse soft tissue edema of the glottis and supraglottic soft tissue causing significant luminal compromise of the larynx and supraglotic airway (red arrows)

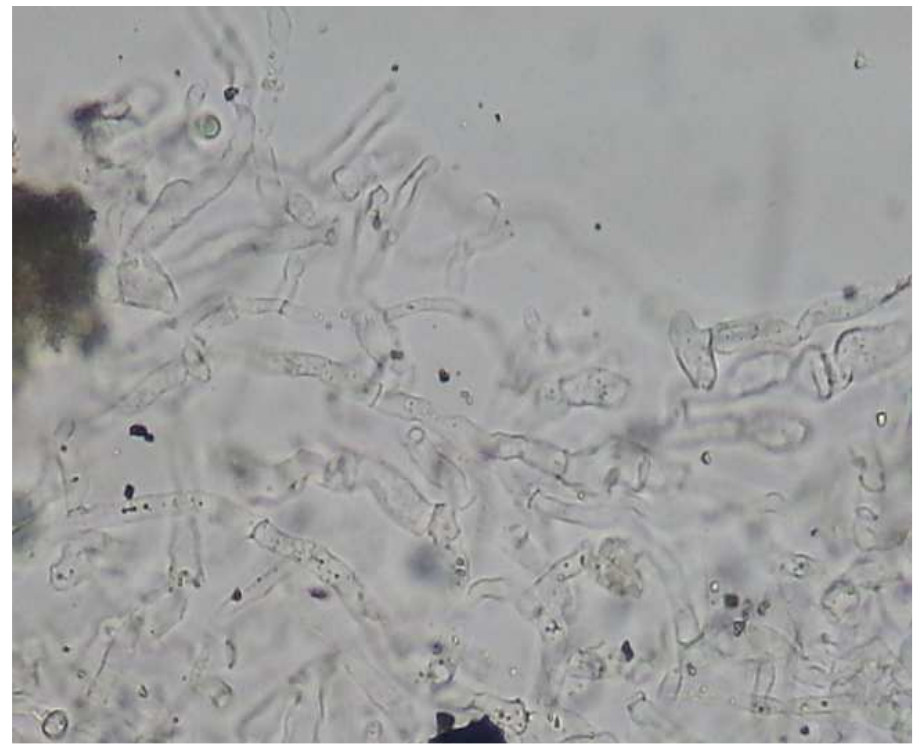

Fig. 2. KOH mount of the bronchoscopy biopsy

As she continued to have stridor and dyspnea despite neutrophil recovery and healing mucositis, fiber optic laryngoscopy was done and biopsy was taken on day +19 . Patient required tracheostomy because of progressive airway obstruction.

\section{Result}

Histopathological evaluation of the laryngeal biopsy specimen revealed chondroid tissue with neutrophilic infiltrate. Gram stain, Z N stain, bacterial cultures and viral Polymerase Chain Reactions (PCRs) from the tissue were negative. There were no viral inclusion bodies seen as well. $\mathrm{KOH}$ stain of the specimen showed thick hyaline, branched, sparsely septate hyphae, suggestive of infection with zygomycetes group of fungus (Fig. 2). She was started on conventional Amphotericin B on day +20 and fungal culture eventually grew Rhizopus species. Posaconazole (400 mg twice daily) was added in view of suboptimal improvement on check laryngoscopy after 2 weeks of Amphotericin therapy. Surgical debridement in the form of partial/total laryngectomy was advised but could not be done because family did not give consent for it. Repeat fiber optic laryngoscopy after a week revealed partial healing with decreasing stenosis. Blockage of tracheostomy tube was tried after 4 weeks of combination antifungal therapy but was not 
tolerated. Immunosuppression tapering was also started from day +50 onwards and we observed falling donor chimerisms as well. Donor chimerism on day +60 was $48 \%$ and she was requiring red cell transfusions every 4 to 6 weeks. She was discharged with tracheostomy tube in situ on oral posaconazole and was planned for a total of 24 to 26 weeks of therapy. Unfortunately, she developed severe bacterial sepsis during follow up and succumbed 5 months post-transplant.

\section{Discussion}

With the widespread use of fluconazole as a prophylactic antifungal agent in HSCT, cases of mucormycosis are increasing (Marr et al., 2000; Sharma et al., 2013). Rhizopus is the most common genus causing human mucormycetes infections in most case series, followed by genera such as Mucor and Lichtheimia, accounting for 70 to $80 \%$ of all mucormycosis cases (Garcia-Vidal et al., 2008). Molds like Rhizopus usually cause localized sinopulmonary infections which can progress to deep seated infections in immunocompromised patients and the outcome of such infections is very poor with very high mortality (Lanternier et al., 2012). Although infection with any mould is associated with high mortality rate, infection with drug-resistant organisms have even poorer outcome (Garcia-Vidal et al., 2008; Lanternier et al., 2012). In our case, patient had deep seated infection of laryngeal cartilage caused by Rhizopus species. Respiratory distress because of progressive airway obstruction becomes a predominant feature in such cases as the disease progresses with the need for a definitive airway in the form of tracheostomy. Significant airway narrowing can often be identified by direct laryngoscopy or radiologically with appropriate cross sectional imaging.

The most commonly implicated mold causing laryngitis is Aspergillus fumigatus and systemic intravenous therapy, in the form of either amphotericin B or voriconazole has been used with some success (Hu et al., 2012; Chandran et al., 2009). Mucormycosis can manifest in different ways. The most common presentation is the rhinocerebral form, involving the nose, paranasal sinuses, orbits and central nervous system. Others organs which can be involved are skin, gastrointestinal, pulmonary and/or as disseminated form. Mucormycosis is an aggressive, frequently fatal invasive fungal infection. Amphotericin and posaconazole have been found to be effective in mucormycosis but surgical debridement is often required for better disease control. High dose corticosteroid therapy results in initial improvement probably due either to attenuation of an allergic upper airways response or reduction in laryngeal oedema. Advanced age, diabetes, long-term steroid therapy, low CD4 lymphocyte cells, leukemia, HIV infection and other causes leading to immunsuppressed state are generally the etiological factors (Liu et al., 2010). The iron overload in thalassemic patients also contributes to increased risk of mucormycosis in such patients (Kontoyiannis and Lewis, 2011).

\section{Conclusion}

Our patient developed histologically proven rhizopus related laryngeal infection requiring tracheostomy with only partial response to combination antifungal therapy. We reviewed the literature and rhizopus has not been so far implicated in causation of laryngeal infection in post stem cell transplant setting. The diagnosis was surely delayed and giving steroids, thinking of reactive laryngeal edema due to engraftment/mucositis, also was counterproductive in our case.

\section{Acknowledgement}

The Authors declare that there is no conflict of interests.

\section{Funding Information}

No funding was received for this manuscript.

\section{Author Contributions}

Mayank Dhamija: Concept, composed original manuscript, literature search, Data and image acquisition, patient care.

Sanjeev K Sharma: Revision of original manuscript, Editions in content and images, Literature search.

Dharma R Choudhary: Patient care, program director, overall guidance.

Vipin Khandelwal: Literature search, Editing.

Gaurav Kharya, Sweta Kothari and Divya Doval: Literature search.

W.V.B.S Ramalingam: Editing, did laryngoscopy, provided images, expert review.

Purabi Burman: Provided microbiology slides and images, expert review.

\section{Conflict of Interest}

None

\section{References}

Chandran, S.K., K.M. Lyons, V. Divi, M. Geyer and R.T. Sataloff et al., 2009. Fungal laryngitis. Ear Nose Throat J., 88: 1026-7. 
Garcia-Vidal, C., A. Upton, K.A. Kirby and K.A. Marr, 2008. Epidemiology of invasive mold infections in allogeneic stem cell transplant recipients: Biological risk factors for infection according to time after transplantation. Clin. Infect. Dis., 47: 1041-1050. DOI: $10.1086 / 591969$

$\mathrm{Hu}, \mathrm{B} ., \mathrm{G} . \mathrm{He}$ and $\mathrm{X}$. $\mathrm{Hu}, 2012$. The aspergillus laryngotracheobronchitis. A case report and literature review. J. Clin. Otorhinolaryngol. Head Neck Surg., 26: 953-955. PMID: 23272504

Kontoyiannis, D.P. and R.E. Lewis, 2011. How I treat mucormycosis. Blood, 118: 1216-1224. DOI: $10.1182 /$ blood-2011-03-316430

Lanternier, F., H.Y. Sun, P. Ribaud, N. Singh and D.P. Kontoyiannis et al., 2012. Mucormycosis in organ and stem cell transplant recipients. Clin. Infect. Dis., 54: 1629-36. DOI: 10.1093/cid/cis195

Liu, Y.C., S.H. Zhou and L. Ling, 2010. Aetiological factors contributing to the development of primary laryngeal aspergillosis in immunocompetent patients. J. Med. Microbiol., 59: 1250-1253.

DOI: $10.1099 / \mathrm{jmm} .0 .021634-0$
Marr, K., K. Seidel, M. Slavin, R.A. Bowden and H.G. Schoch et al., 2000. Prolonged fluconazole prophylaxis is associated with persistent protection against candidiasis-related death in allogeneic marrow transplant recipients: Long-term follow-up of a randomized, placebo-controlled trial. Blood, 96: 2055-61. PMID: 10979947

Marr, K.A., R.A. Carter, F. Crippa, A. Wald and L. Corey, 2002. Epidemiology and outcome of mould infections in hematopoietic stem cell transplant recipients. Clin. Infect. Dis., 34: 909-17. DOI: $10.1086 / 339202$

Sharma, S.K., S. Kumar, A.K. Singh, T. Seth and P. Mishra et al., 2013. Feasibility and outcome of CT-guided lung biopsy in patients with hematological diseases and suspected fungal pneumonia. J. Infect. Dev. Ctries, 7: 748-752. DOI: $10.3855 /$ jidc. 2823 